demands of the Deutsche Geodätische Kommission and is also necessary for the special needs of astronomy itself. The number of bright stars in $\mathrm{FK}_{3}$ and $\mathrm{FK}_{3}$ Supp. is nearly the same as the number of bright stars in N30. But the distribution of these stars in both hemispheres is much more regular than in $N_{30}$.

Positions and proper motions of $\mathrm{FK}_{3}$ Supp. stars are at the moment taken from $\mathrm{N}_{30}$. But it is absolutely necessary to observe in future these stars on the meridian circles of both hemispheres. I therefore propose to observe these Supp. stars within the programme of the Anhaltssterne for AGK 3, as it is also planned for other stars. Observers who wish to observe $\mathrm{FK}_{3}$ Supp. should give a note to the Astronomisches Rechen-Institut.

REMARKS

Answering a question from Prof. Heckmann, Prof. Kopff explained that he was willing to provide to observers intermediate corrections for 1957 as well as for later dates.

\title{
2. FK3, N3o AND PULKOVO ABSOLUTE CATALOGUES
}

\author{
By A. A. Nemiro
}

\section{Pulkovo absolute observations}

The Pulkovo absolute catalogues of right ascensions and declinations of stars have played an important part in deriving the fundamental systems now in use. The Pulkovo catalogues were given very high weights when the work on the $\mathrm{FK}_{3}$, the GC and the $\mathrm{N}_{30}$ was carried out.

The continuation of the traditional Pulkovo series of absolute determinations appears to be a very important task at the present time too, since this work is closely connected with the improvement of the existing fundamental systems.

Another work of paramount importance is the preparation of the new fundamental catalogue of faint stars (FKSZ), the proper motions of which must be referred to extragalactic nebulae.

The preparation of the FKSZ has a direct bearing on the classical problem of the formation of fundamental systems. That is why the meridian observations of brighter and fainter stars should be carried out simultaneously with the same instruments.

In view of these two requirements, the Pulkovo programme of absolute observations has been considerably extended. It contains now not only the bright Pulkovo stars, but also all the FKSZ stars in the zone from $+90^{\circ}$ to $-10^{\circ}$.

The list of bright stars involved in the programme of absolute determinations was revised(r). Some double stars, as well as some stars for which neighbouring stars might impede visual and especially photo-electric observations, were replaced. The present programme thus covers 625 bright fundamental stars in the declination zone from $+90^{\circ}$ to $-30^{\circ}$. $5 \mathrm{I} 5$ of them (from $+90^{\circ}$ to $-10^{\circ}$ ) are observed at Pulkovo. The stars are uniformly distributed in $\alpha$ and $\delta$ (but not over the area!). All of them are contained in the FK3. In this way, with an additional 53I FKSZ stars, the Pulkovo programme of absolute observations covers I046 stars, 332 of them being observed in both culminations. This new Pulkovo programme has also been adopted at Nikolaiev, Tashkent and Kazan.

In our opinion, the number of stars in absolute catalogues must not exceed ca. I000, since such a limitation contributes to the improvement of the accuracy of the observations. The observations for catalogues containing several thousands of stars can be performed quite efficiently with meridian circles by means of differential methods.

The Pulkovo methods of absolute determinations of right ascensions and declinations have been employed quite successfully for many decades both at Pulkovo and at some other observatories. At present some modifications of these methods are required. In determining the right ascensions, it seems advisable, for instance, to confine the observation of stars to night time. This is particularly important for the photo-electric registration of star transits which will be made in the very near future. With this aim in view, a new 
chain method of determining the absolute azimuth has been devised at Pulkovo, by which day-time observations are avoided(2). This method is now under examination. The number of clock stars is now I47 (against 30 in the old programmes). Their right ascensions will be smoothed by a method similar to the conventional Wagner's method which combines the clock stars into several groups. It was thought inadvisable to represent the required corrections in the form of a trigonometrical series, as the nature of the terms $\Delta \alpha_{\alpha}$ is more complicated.

The collimation of the large transit instrument is determined every night by means of two collimators, without reversing the instruments. The pivots of this instrument have been carefully examined by various methods. The most accurate results have been obtained with the aid of Ouverky's interferometer. A complete scrutiny of pivots with this device ( 6 series per every $5^{\circ}$ ) takes about $4 \mathrm{hr}$., the mean error of the final results being between $+0.01 \mu$ and $\pm 0.02 \mu$. The thermal effects of the instrument are being studied.

The clock corrections and rates, as determined with the large transit instrument, are compared with the data of the Time Service, a fair control of the instrument system being thus obtained.

Preparations are made for introducing the photo-electric registration of star transits for absolute determinations of right ascensions.

As far as the determination of declinations is concerned, G. K. Zimmerman (3) and B. A. Orlov (4) have already proved that the method of eliminating flexure by replacing the object-glass and the eyepiece is more or less invalid. Accordingly, a special investigation of the flexure of the vertical circle at different zenith distances has been undertaken at Pulkovo using the original method proposed by V. P. Linnik, based upon V. A. Eliastratov's idea of using for this purpose a collimator fixed at different zenith distances (5).

The errors of the vertical circle divisions have been investigated in detail.

The day-time meridian observations will be carried out at Pulkovo for determinations of the zero-point corrections to the right ascensions $(\Delta \mathrm{A})$ and to the equator $\left(\Delta \delta_{0}\right)$ from observations of the Sun. Since observations of the Sun at Pulkovo are practically impossible in winter, they will be effected only during the periods March-April and September-October, symmetrically with respect to the equinoxes.

The analysis of the Pulkovo observations of the Sun shows that the limitation of observations to the vernal and autumnal periods (near the equinoxes) does not appreciably decrease the accuracy of $\Delta \mathrm{A}$ and $\Delta \delta_{0}$ values. Under these conditions it is impossible to determine the correction to the obliquity of the equator in respect to the ecliptic; but the study of this problem at Pulkovo is generally impractical.

Systematic observations of the Sun, Moon and planets are carried out throughout the year at the Nikolaiev branch of the Pulkovo Observatory. These observations will also be used for $\Delta \mathrm{A}$ and $\Delta \delta_{0}$ determinations of the Pulkovo absolute catalogues. The correction to the equinox will be derived independently from the photographic observations of minor planets at Pulkovo and at other observatories, in accordance with the KSZ programme.

The absolute observations begun in I 955 will be completed in I959 and the catalogues published in I 960 .

Absolute observations of 625 bright stars will be started soon at Nikolaiev. The FKSZ stars cannot be observed at Nikolaiev on account of the moderate size of the Nikolaiev transit instrument and the vertical circle (aperture $=108 \mathrm{~mm}$.).

2. The Pulkovo fundamental catalogue of Right ascensions

In the course of recent years a discussion of the Pulkovo absolute catalogues has been carried out at Pulkovo.

B. A. Orlov has investigated the catalogues of declinations, while the present author has examined the catalogues of right ascensions.

As a result of the latter investigation it proved possible to utilize the Pulkovo observations of right ascensions for compiling an independent fundamental catalogue (Pu $\alpha \mathrm{I})$. 
A sufficient stability of the transit instrument system, particularly with regard to errors $\Delta \alpha_{\alpha}(6,7)$ favoured such a design. For deriving the fundamental system of right ascensions of the $\mathrm{Pu} \alpha \mathrm{I}$ the following 9 Pulkovo catalogues were employed: $\mathrm{r} 845$, I865, I885, I892, I900, I905, I9I5, I925, and I930. The weights assigned to these catalogues were: I845, $0.25 ; 1865,0.8 ; 1885, I 892$ and $1900,0.6$; the remaining catalogues, $I \cdot 00$.

The Pulkovo observations of the Sun for computing the correction to the equinox were not used, and the equinox of the $\mathrm{FK}_{3}$ was retained.

The mean epoch of the $\mathrm{Pu} \alpha \mathrm{I}$ is I902.0.

The comparison of the $\mathrm{Pu} \alpha \mathrm{I}$ with the $\mathrm{FK}_{3}$ and the $\mathrm{N}_{3}$ o with respect to $\Delta \alpha_{\delta}$ for I9O2 and $\Delta \mu_{\delta}$ (centennial) in $\mathrm{O}^{\mathrm{s}} \cdot \mathrm{OOI}$ is illustrated in Table $\mathrm{I}$.

The values of $\Delta \alpha_{\delta}$ and $\Delta \mu_{\delta}$ for $\mathrm{Pu} \alpha \mathrm{I}-\mathrm{N} 30$ have been computed by adding to the differences $\mathrm{Pu} \propto \mathrm{I}_{-}-\mathrm{FK}_{3}$ the respective differences $\mathrm{FK}_{3}-\mathrm{N}_{30}$, which we obtained by using only the fundamental stars of the $\mathrm{FK}_{3}$. A separate comparison of the fundamental Auwers Sterne and the supplementary $\mathrm{FK}_{3}$ catalogues (ZS) with $\mathrm{N}_{30}$ has shown that between the systems of the fundamental and the supplementary stars there are small, but definitely appreciable discrepancies (see Table 2). Similar discrepancies have been revealed in the errors $\Delta \alpha_{\alpha}$ and $\Delta \mu_{\alpha}$.

Table I

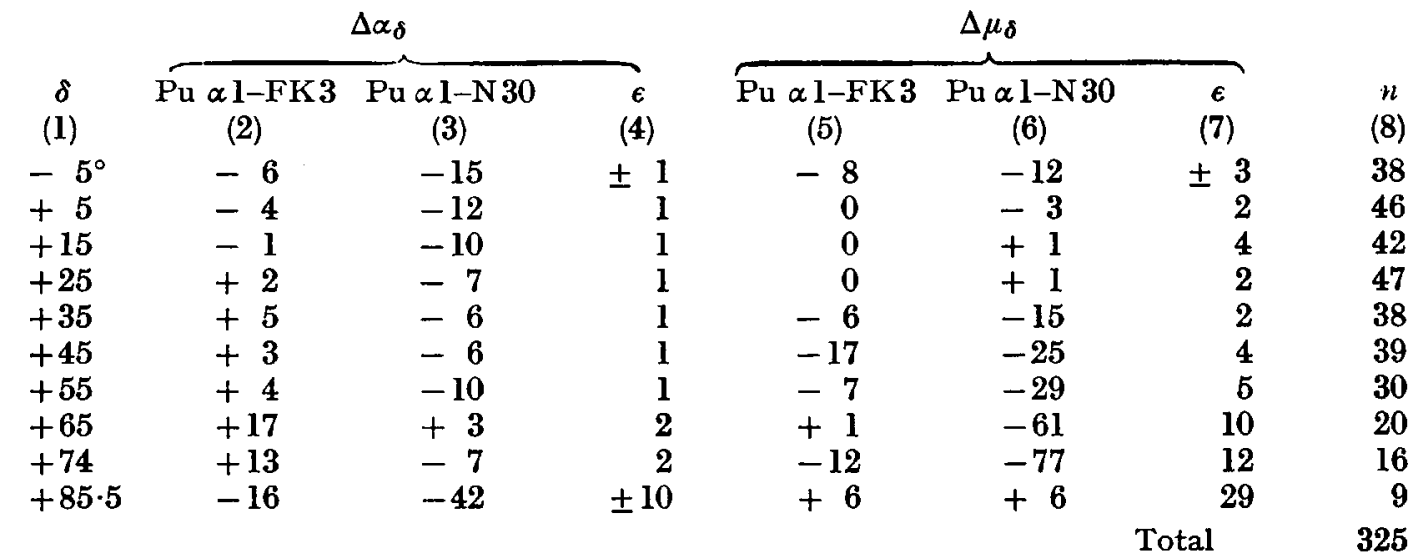

Table 2

\begin{tabular}{|c|c|c|c|c|c|c|}
\hline \multirow[b]{2}{*}{$\delta$} & \multicolumn{3}{|c|}{$\Delta \alpha_{1950.0}$ in $0^{8} .001$} & \multicolumn{3}{|c|}{$\Delta \mu$ (centennial) in $0^{8} .001$} \\
\hline & $\begin{array}{c}\mathrm{N} 30-\mathrm{FK} 3=\mathrm{I} \\
\text { (Fund. list) }\end{array}$ & $\begin{array}{c}\text { N 30-FK3 = II } \\
\text { (Suppl. list) } \\
\text { (ZS) }\end{array}$ & II-I & $\begin{array}{c}\text { N30-FK3 = I } \\
\text { (Fund. list) }\end{array}$ & $\begin{array}{c}\text { N 30-FK3=II } \\
\text { (Suppl. list) } \\
\text { (ZS) }\end{array}$ & II-I \\
\hline$-5^{\circ}$ & +11 & +15 & +4 & +4 & +16 & +12 \\
\hline+5 & +9 & +11 & +2 & +3 & +5 & +2 \\
\hline+15 & +9 & +13 & +4 & -1 & +7 & +8 \\
\hline+25 & +9 & +7 & -2 & -1 & -3 & -2 \\
\hline+35 & +15 & +15 & 0 & +9 & +10 & +1 \\
\hline+45 & +13 & +10 & -3 & +8 & +6 & -2 \\
\hline+55 & +25 & +17 & -8 & +22 & +12 & -10 \\
\hline+65 & +45 & +37 & -8 & +62 & +57 & -5 \\
\hline+75 & +52 & +44 & -8 & +65 & +34 & -31 \\
\hline+85 & +26 & +3 & -23 & 0 & -70 & -70 \\
\hline
\end{tabular}

The data of Table 3 represent a comparison of the $\mathrm{Pu} \alpha \mathrm{I}$ and the $\mathrm{N} 30$ with the $\mathrm{FK}_{3}$ with respect to the errors $\Delta \alpha_{\alpha}$ and $\Delta \mu_{\alpha}$ (centennial) in $0^{8}$-oor. It may be seen from the figures of Table 3 that up to declination $40^{\circ}$ systematic discrepancies between the $\mathrm{FK}_{3}$, $\mathrm{N} 30$ and $\mathrm{Pu} \propto \mathrm{I}$ are small. In the zone $+40^{\circ}-+60^{\circ}$ these differences increase considerably and in the zone $+60^{\circ}-+80^{\circ}$ they become quite appreciable, the variation of the differences $\mathrm{Pu} \propto \mathrm{I}_{-}-\mathrm{FK}_{3}$ and $\mathrm{N}_{30}-\mathrm{FK}_{3}$ being in fair agreement. We are thus led to the 
suggestion that in the zone $+40^{\circ}-+80^{\circ}$ the $\mathrm{FK}_{3}$ is affected by considerable systematic errors of the form $\Delta \alpha_{\alpha}$ and $\Delta \mu_{\alpha}$. This conclusion has been confirmed by N. N. Pavlov, who has published the $\Delta \alpha_{\alpha}$ corrections to the $\mathrm{FK}_{3}$ for I950, as derived from photoelectric observations with the transit instrument of the Time Service (8).

We have deemed it necessary to call attention to the above errors in the $\mathrm{FK}_{3}$, and hope that our results may be of use for the revision of the $\mathrm{FK}_{3}$ and the compilation of the $\mathrm{FK}_{4}$ now in progress at Heidelberg under the supervision of Prof. Kopff.

The fair agreement between the $\mathrm{Pu} \alpha \mathrm{I}$ and the $\mathrm{N} 30$ with respect to $\Delta \alpha_{\alpha}$ and $\Delta \mu_{\alpha}$ indicates the high precision of both systems. It should be mentioned that the method of deriving the proper motions from the differences of star positions referred to two epochs, as employed in the $\mathrm{N}_{30}$, is sometimes more advantageous as compared to the conventional method of determining proper motions. Its advantage consists in a more thorough elimination of systematic errors, which compensates the decrease in the weight of the results, since in the proper motion determinations the systematic errors of catalogues are more important than the accidental errors.

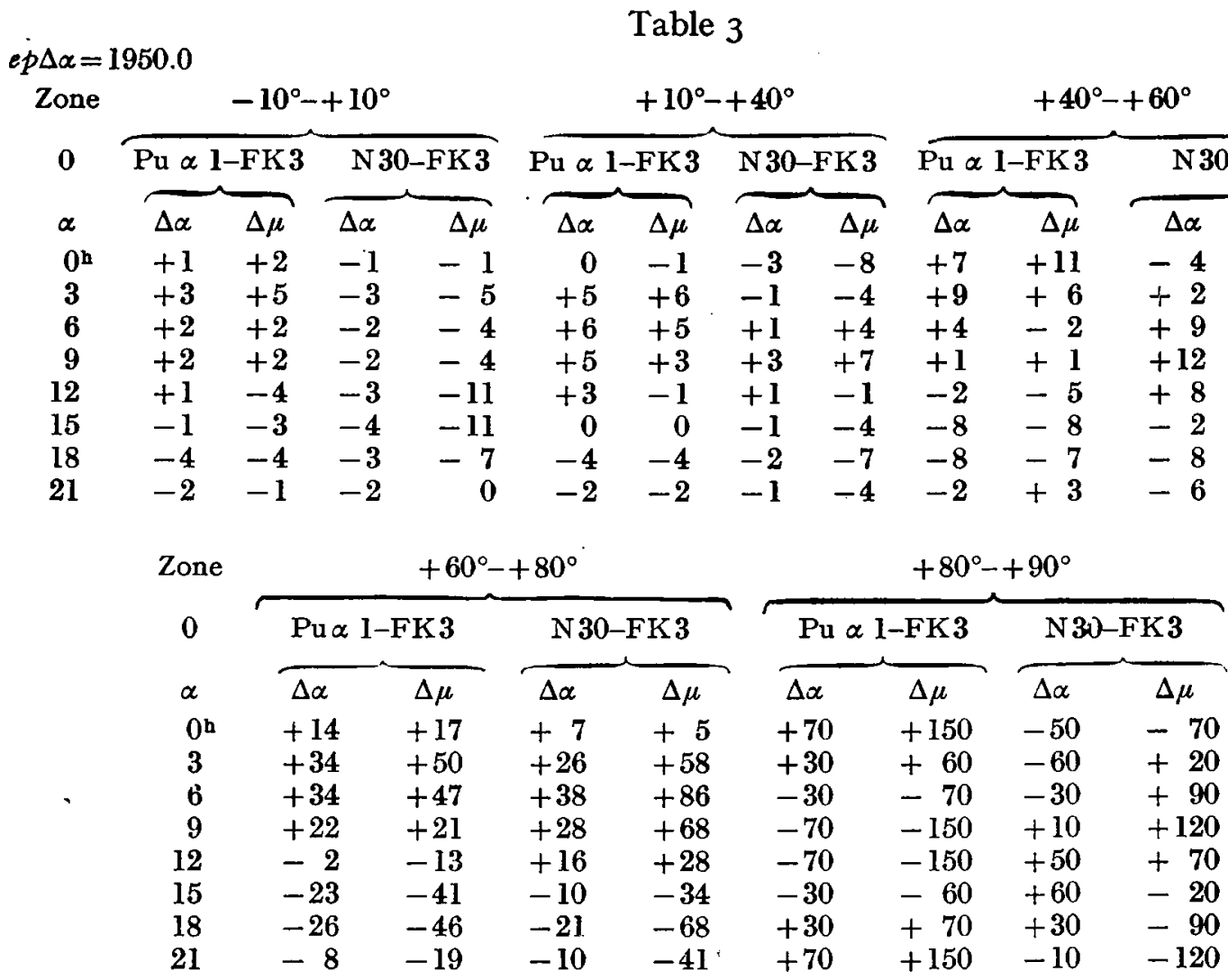

\section{SUMMARY}

(I) Prolonged series of observations with the same instrument are extremely valuable for deriving systems of fundamental catalogues.

(2) A regular exchange of detailed information on the up-to-date methods of absolute determinations, employed at different observatories, appears to be highly desirable.

(3) It seems advisable that along with the bright stars, the programmes of absolute observations should contain faint stars as well (FKSZ), because the latter permit direct reference to the extragalactic nebulae and minor planets. 


\section{REFERENCES}

(I) M. S. Zverev, A. A. Nemiro, K. N. Tavastsherna, Trans. I Ith Astrometrical Conference, U.S.S.R. 1955 .

(2) A. A. Nemiro, Pulkovo Bull. no. I43, I950.

(3) G. K. Zimmerman, Pulkovo Bull. no. 143, 1950.

(4) B. A. Orlov, Pulkovo Bull. no. I50, I953.

(5) V. A. Elistratov, A.J. U.S.S.R. I7, no. 2, 1940.

(6) A. A. Nemiro, Trans. I ith Astrometrical Conference, U.S.S.R. I955.

(7) A. A. Nemiro, Astron. Circular, U.S.S.R. no. I59, 1955.

(8) N. N. Pavlov, Sprawozdania Polskiego Towarzystwa Astronomisznego, Zescyt III, I951.

\section{REMARKS}

Dr Fricke: The catalogue $\mathrm{Pu} \propto \mathrm{I}$ will be used for the systematic revision of the $\mathrm{FK}_{3}$ if forwarded to the Astronomische Rechen-Institut before July I956. As to a remark by $\mathrm{Dr}$ Nemiro that $\mathrm{Pu} \alpha \mathrm{I}$ is in good agreement with $\mathrm{N} 30$, attention is drawn to the differences $\Delta \mu_{\alpha}$ when comparing $\mathrm{Pu} \alpha \mathrm{I}, \mathrm{N}_{30}$ and $\mathrm{FK}_{3}$. These differences show that the accidental and systematic deviations of $\mathrm{Pu} \propto \mathrm{I}$ from $\mathrm{N}_{30}$ and FK 3 are remarkably large.

\section{MERIDIAN WORK AT THE ROYAL GREENWICH OBSERVATORY By H. SPEncer Jones}

The fundamental meridian work at the Greenwich Observatory during the past century has been undertaken with the Airy transit circle, which was installed in I85I. This instrument, designed in all its details by Airy, is a massive non-reversible instrument with an 8 in. objective. Though in advance of its time when it was designed it does not meet modern requirements, but the long series of observations made with the same instrument have made an important contribution to fundamental positional astronomy.

The housing of the instrument, in a pavilion with buildings on each side and in which the observer is in a pit, is such that refraction anomalies are to be expected. The telescope was provided with a movable mercury horizon so that stars at various altitudes could be observed by reflexion, as a control on instrumental flexure. Many papers were written on the discordances between direct and reflected observations, and it is certain that refraction anomalies were an important contributory factor to the (R-D) discordances.

The telescope was supported by its pivots in two long bearings, the bearings being carried by stone piers. No adjustments for level and azimuth were provided: Airy stated that none would be needed. In I92I, after a very dry summer, the east pier started to sink relatively to the west pier. This sinking has since continued at a fairly uniform rate, and it became necessary to pack up the east bearing each year. The bearings were modified in I923 as it had proved impossible to maintain precise alignment of the original long-bearing surfaces.

Observations were made at first by the hand tapping method; the relative personal equations of the observers were determined and the observations of transits were reduced to the system of a standard observer. In I9I 5 a hand driven travelling wire micrometer was substituted and it was then found that transits were recorded about $0.25 \mathrm{sec}$. early by the standard observer.

The site of the instrument was not ideal. The ground falls away steeply to the north and less steeply to the south. It was thus not possible to provide azimuth marks for the control of changes in azimuth. About I830 an azimuth mark on the Bradley meridian ( $\mathrm{g} \mathrm{ft}$. to the west of the meridian of the Airy transit circle, which defines the zero meridian of longitude) had been erected at Chingford to the north of London. This mark had long been unobservable, but recently it was observed for a period with the Airy instrument by placing a light on it, and the observations have shown that the Airy instrument has an appreciable diurnal variation of azimuth. 